\title{
ON SCATTERED COMPACT ORDERED SETS
}

\author{
GADI MORAN
}

\begin{abstract}
A compact ordered set $K$ enjoys the property that every continuous real function on $K$ is a sum of its increments if and only if $K$ is scattered.
\end{abstract}

A topological space $K$ is said to be scattered if every nonempty subset of $K$ contains a relatively isolated point. Let $C(K)$ denote the Banach space of real continuous functions on a compact Hausdorff space $K$, with the supremum norm. In [PS] Pelcziński and Semadeni characterize the scattered compact Hausdorff spaces $K$ in the class of compact Hausdorff spaces by isomorphism invariants of $C(K)$, among other characterizations (see also [BP]). The purpose of this note is to characterize the class of scattered compact orders among the compact orders by means of properties of their continuous real valued functions as follows.

TheOREM 1. Let $K$ be a nonempty compact ordered set. Then the following conditions are equivalent:

(a) $K$ is scattered;

(b) every $f \in C(K)$ is a sum of its increments;

(c) every $f \in C(K)$ is uniquely determined by its increments;

(d) for every $A \subset K,|\bar{A}|=|A|$.

A short dictionary and few remarks are in order. $\bar{A}$ denotes the closure of $A \subseteq K$, and $|A|$ denotes the cardinality of $A$. Given an ordered set $K$ and $a \in K$, define the right neighbor $\mathrm{rn}_{K}(a)$ of $a$ in $K$ as the greatest $b \in K$ such that no $c \in K$ satisfies $a<c<b$.

Before going on it is useful to recall:

Proposition 1 ([HK], see [K p. 162]). Let $K$ be an ordered set. Then $K$ is compact iff every nonempty subset of $K$ has a least upper bound and a greatest lower bound.

Let $m_{K}\left[M_{K}\right]$ denote the minimal [maximal] element of the nonempty compact ordered set $K$. Let $f \in C(K)$. Define the increment-derivative $f^{\dagger}$ of $f$ by $f^{\dagger}(a)=f\left(\mathrm{rn}_{K}(a)\right)-f(a)(a \in K)$. Note that by compactness of $K$ and continuity of $f$, the set $\left\{a \in K:\left|f^{\dagger}(a)\right|>\varepsilon\right\}$ is finite for every $\varepsilon>0$, and so

Received by the editors May 16, 1978 and, in revised form, August 22, 1978.

AMS (MOS) subject classifications (1970). Primary 54F05, 54D30, 46E15; Secondary 54C30, 06A05, 46E15.

Key words and phrases. Scattered compact ordered set, continuous real functions, increments. 
$f^{\dagger}$ has a countable support. Let the a-increment of $f$ be the function $J \in$ $C(K)$ defined by $J(b)=0$ for $b<a$ and $J(b)=f^{\dagger}(a)$ for $b>a$. For a real number $r$ let $r^{K} \in C(K)$ be the constant function with value $r$ everywhere. We shall write $r$ instead of $r^{K}$ when no confusion may arise. $J \in C(K)$ is called an increment of $f$ if $J$ is an $a$-increment of $f$ for some $a \in K$, or if $J=f\left(m_{K}\right)$. Since $f^{\dagger}$ has a countable support, $f$ has only countably many increments. We say that $f$ is a sum of its increments if $f=\Sigma_{n} J_{n}$ holds in $C(K)$, where $\left(J_{n}\right)_{n}$ is an enumeration of $f$ 's increments. $f \in C(K)$ is uniquely determined by its increments iff $\tilde{f} \in C(K), \tilde{f}^{\dagger}=f^{\dagger}, \tilde{f}\left(m_{K}\right)=f\left(m_{K}\right)$ imply $\tilde{f}=f$. Note that by $(\tilde{f}-f)^{\dagger}=\tilde{f}^{\dagger}-f^{\dagger}$, (c) is equivalent to

$\left(c^{\prime}\right) f\left(m_{K}\right)=0$ and $f^{\dagger}=0$ imply $f=0(f \in C(K))$.

Let $Q_{0}$ denote the ordered set of rational numbers in the closed unit interval $[0,1]$. Consider the following statement on an ordered set $K$ :

(a') $K$ does not contain a subset order isomorphic to $Q_{0}$.

(a') implies (a): In fact assume that $K$ is not scattered, and let $D_{0} \subseteq K$ be a nonempty set with no relatively isolated points. Notice that if $a, b \in D_{0}$, $b=\mathrm{rn}_{D_{0}}(a)$ and $a<b$, then $\operatorname{rn}_{D_{0}}(b)=b$ (otherwise $b$ is isolated in $D_{0}$ ). Removing from $D_{0}$ those points $a$ for which $a<\mathrm{rn}_{D_{0}}(a)$ we obtain a subset $D_{1}$ of $D_{0}$ such that: for any $a, b \in D_{1}$ with $a<b$ there is a $c \in D_{1}$ with $a<c<b$. It is now easy to pick a subset $D \subseteq D_{1}$ which in addition is countable and has a first and last element. Such a set is well known to be order isomorphic with $Q_{0}$.

If $K$ is compact, then (a) implies ( $\left.\mathrm{a}^{\prime}\right)$ as well. Indeed, let $D \subseteq K$ be order isomorphic to $Q$. By Proposition 1, every subset of $D$ has a supremum in $K$, and so $\bar{D}$ has the cardinality of the continuum, contradicting (d). The compactness assumption is necessary for this implication: replace every rational number by a copy of the set of integers. The ordered set obtained is scattered-as a matter of fact, discrete-but contains a subset order isomorphic with $Q_{0}$.

We refer the reader to $[\mathbf{M}]$ for other notation used, and for a proof of:

(e) Let $K$ be a countable compact ordered set. Then every $f \in C(K)$ is a sum of its increments.

Proof of TheOREM 1. Our proof of Theorem 1 consists of the following implications:

(1) (b) $\Rightarrow$ (c),

(2) (c) $\Rightarrow$ (a), (d) $\Rightarrow$ (a),

(3) (a) $\Rightarrow$ (c), (a) $\Rightarrow$ (d),

(4) (c) + (d) + (e) $\Rightarrow$ (b).

(1) (b) $\Rightarrow$ (c): Assume (b) and let $f \in C(K)$ satisfy $f\left(m_{K}\right)=0, f^{\dagger}=0$. Then 0 is the only increment of $f$, and so, by (b), $f=0$. Thus ( $\left.c^{\prime}\right)$ holds, hence (c).

(2) (c) $\Rightarrow$ (a), (d) $\Rightarrow$ (a). Assume not (a), hence not (a'). Let $D \subseteq K$ be order isomorphic to $Q_{0}$, and let $g: D \rightarrow Q_{0}$ be an order isomorphism. By 
Proposition $1,|\bar{D}|=2^{\kappa_{0}}$, hence (d) fails. Define a real valued $f$ on $K$ by $f(a)=0$ for $a \leqslant m_{D}$, and $f(a)=\sup \{g(d): d \in D, d<a\}$ for $m_{D}<a$. It is readily checked that $f \in C(K)$ and $f$ maps $K$ onto the unit interval $[0,1]$. But $f\left(m_{K}\right)=0$ and $f^{\dagger}=0$, and so $\left(c^{\prime}\right)$, hence (c), fails.

(3) (a) $\Rightarrow$ (c), (a) $\Rightarrow$ (d). We summarize some notions and facts from the theory of scattered spaces first. Let $X^{\prime}$ denote the set of nonisolated points in the topological space $X$. Define $X^{(\mu)}$ for every ordinal $\mu$ by $X^{(0)}=X$, $X^{(\mu+1)}=X^{(\mu)^{\prime}}$ and $X^{(\nu)}=\cap_{\mu<\nu} X^{(\mu)}$ for a limit ordinal $\nu$. Then $X^{(\mu)}$ is closed in $X$ and $X^{(\mu)} \supseteq X^{(v)}$ for $\mu<\nu$. Let $\operatorname{rk}(X)$ be the smallest $\nu$ such that $X^{(v)}=X^{(\nu+1)}$. Then $X$ is scattered iff $X^{(v)}=\varnothing$ for $\nu \geqslant \operatorname{rk}(X)$. If $X$ is a scattered compact Hausdorff space then $\operatorname{rk}(X)=\mu+1$ and $X^{(\mu)}$ is finite. Define $\operatorname{ch}(X)$ for a scattered compact Hausdorff space $X$ by $\operatorname{ch}(X)=(\mu, m)$, where $\operatorname{rk}(X)=\mu+1$ and $\left|X^{(\mu)}\right|=m$.

Proposition 2. Let $K$ be a scattered ordered set, and let $a, b \in K, a<b$. If $(a, b) \neq \varnothing$ then there is an isolated point $c \in K$ with $a<c<b$.

Proof. Indeed, if the open interval $(a, b)$ is nonempty, it contains a relatively isolated point $c$. Since $(a, b)$ is open in $K, c$ is an isolated point of $K$.

By Proposition 2 and its preceding remarks we have:

Proposition 3. Let $K$ be a nonempty scattered compact ordered set and let $\operatorname{ch}(K)=(\mu, m)$. Then $K=\sum_{i<m} K_{i}$ where $K_{i}$ is a scattered compact ordered set satisfying $\operatorname{ch}\left(K_{i}\right)=(\mu, 1)(i<m)$.

Let $A, B$ be subsets of $K$. We say that $A$ is upper cofinal [lower cofinal] in $B$ iff for every $b \in B$ there is an $a \in A$ such that $b \leqslant a[a \leqslant b]$. We also agree that $\varnothing$ is (upper and lower) cofinal in $\varnothing$. Given $a \in K$, define cf_(a) $\left[\mathrm{cf}_{+}(a)\right]$ to be the least ordinal $\rho$ such that $\left[m_{K}, a\right)$ contains an upper cofinal subset order isomorphic with $\rho$ (the least ordinal $\sigma$ such that $\left(a, M_{K}\right.$ ] contains a lower cofinal subset order isomorphic with the inverse order of $\sigma$ ). Then $\mathrm{cf}_{-}(a), \mathrm{cf}_{+}(a)$ are actually regular cardinals, $\mathrm{cf}_{-}\left(m_{K}\right)=\mathrm{cf}_{+}\left(M_{K}\right)=0, \mathrm{cf}_{-}(a)$ $=1\left[\mathrm{cf}_{+}(a)=1\right]$ iff $a=\mathrm{rn}_{K}(b)$ for $b<a\left[b=\mathrm{rn}_{K}(a)\right.$ for $\left.a<b\right]$ and in other cases cf_(a), $\mathrm{cf}_{+}(a)$ are infinite regular cardinals. Given $a \in K$ with cf $(a)>1$ we may by Proposition 2 pick in $\left[m_{K}, a\right)$ an upper cofinal set of order type cf_(a) consisting entirely of isolated points. An analogous statement is true of $\left(a, M_{K}\right]$. Hence we have:

Proposition 4. Let $K$ be a scattered compact ordered set, $a \in K$, and let $\rho=\mathrm{cf}_{-}(a), \sigma=\mathrm{cf}_{+}(a)$. Then there are sequences $\left(L_{\gamma}\right)_{\gamma<\rho},\left(U_{\delta}\right)_{\delta<\sigma}$ of nonempty closed intervals in $K$ such that:

(1) $L_{\gamma}<L_{\gamma^{\prime}}<a<U_{\delta^{\prime}}<U_{\delta}\left(\gamma<\gamma^{\prime}<\rho, \delta<\delta^{\prime}<\sigma\right)$,

(2) $\left[m_{k}, a\right)=\bigcup_{\gamma<\rho} L_{\gamma},\left(a, M_{K}\right]=\bigcup_{\delta<\sigma} U_{\delta}$.

We are now ready to prove

(3) $\quad$ (a) $\Rightarrow$ (d) (a) $\Rightarrow$ (c). 
Proposition 5. Let $K$ be a scattered compact ordered set. Then:

(i) if $A \subseteq K$ then $|\bar{A}|=|A|$;

(ii) if $f \in C(K)$ satisfies $f\left(m_{k}\right)=0$ and $f^{\dagger}=0$ then $f=0$.

Proof. We prove (i) and (ii) by induction on $\operatorname{ch}(K)=(\mu, m)$. (i) and (ii) are true for finite $K$ so we may assume $0<\mu$. If $K=K_{1}+K_{2}$, where $K_{1}$ and $K_{2}$ are compact ordered sets for which (i) and (ii) holds, then (i) and (ii) holds of $K$ as well. Hence, by Proposition 3, we may assume that $\operatorname{ch}(K)=(\mu, 1)$, and that (i) and (ii) are true for all compact ordered sets $K^{\prime}$ with $\operatorname{ch}\left(K^{\prime}\right)=$ $\left(\mu^{\prime}, m\right)$ where $\mu^{\prime}<\mu$ and $m \in \omega$. Assume then that $K^{(\mu)}=\{a\}$, and let $\rho=\mathrm{cf}_{-}(a), \sigma=\mathrm{cf}_{+}(a)$. Let $\left(L_{\gamma}\right)_{\gamma<\rho},\left(U_{\delta}\right)_{\delta \in \sigma}$ be sequences of nonempty closed intervals of $K$ satisfying (1) and (2) of Proposition 4. Then $L_{\gamma}, U_{\delta}$ are compact ordered sets satisfying $L_{\gamma}^{(\nu)}, U_{\delta}^{(\nu)} \subseteq K^{(\nu)}$ for every ordinal $\nu$. Hence $L_{\gamma}^{(\mu)} \subseteq L_{\gamma} \cap K^{(\mu)}$ whence $L_{\gamma}^{(\mu)}=\varnothing$. Similarly, $U_{\delta}^{(\mu)}=\varnothing$ and so we may assume that for each $\gamma<\rho, \delta<\sigma$, (i) and (ii) are true of $L_{\gamma}, U_{\delta}$.

We next prove that (i) is true of $K$. First note that (i) is true of an arbitrary well-ordered set $D$. In fact, let $A \subseteq D$ where $D$ is well-ordered. Then $\bar{A}$ is well-ordered, and so let $g$ be an order preserving mapping of $\bar{A}$ onto an ordinal $\lambda$. Let $B$ denote the set of limit ordinals smaller than $\lambda$. Then $|\lambda|=|\lambda-B|$ and $\lambda-B \subseteq g(A) \subseteq g(\bar{A})=\lambda$, and so $|\lambda|=|\lambda-B|<|g(A)|$ $=|A| \leqslant|\bar{A}|=|g(\bar{A})|=|\lambda|$, whence $|A|=|\bar{A}|$.

Now let $A \subseteq K$, and we show that $|A|=|\bar{A}|$. Let $A_{-}=A \cap\left[m_{k}, a\right)$, $A_{+}=A \cap\left(a, M_{K}\right]$. Since $\bar{A} \subseteq \bar{A}_{-} \cup\{a\} \cup \bar{A}_{+}$, it is enough to prove (i) for $A_{-}$and for $A_{+}$. Thus, we shall assume $A \subseteq\left[m_{k}, a\right)$. The other case is proved similarly. Since (i) is obvious for finite $A$, we further assume that $A$ is infinite.

Let $A_{\gamma}=A \cap L_{\gamma}(\gamma<\rho)$, let $D=\left\{\gamma<\rho: A_{\gamma} \neq \varnothing\right\}$, and let $\bar{D}$ denote the closure of $D$ in $\rho$. Let $m_{\gamma}=m_{L_{\gamma}}$, and define $E \subseteq K$ by

$$
E=\{a\} \cup\left\{m_{\gamma}: \gamma \in \bar{D}\right\} \cup \bigcup_{\gamma \in D} \overline{A_{\gamma}} .
$$

Now, by definition $|D| \leqslant|A|$ and $\left|A_{\gamma}\right| \leqslant|A|(\gamma<\rho)$. Hence by the wellordered case, $|\bar{D}|=|D| \leqslant|A|$ and by the induction hypothesis $\left|\bar{A}_{\gamma}\right|=\left|A_{\gamma}\right|<$ $|A|$. Thus, $|E| \leqslant|A|+|A|^{2}+1$ and since $A$ is infinite, $|E|<|A|$. Also, $A \subseteq E$, and it is easily checked that $E$ is closed in $K$. Thus, $\bar{A} \subset E$, whence $|\bar{A}|<|E|<|A|$ and so $|\bar{A}|=|A|$. Thus, (i) is true of $K$.

Finally, we prove that (ii) is true of $K$. Let $f \in C(K)$ satisfy $f\left(m_{k}\right)=0$ and $f^{\dagger}=0^{K}$. Let $m_{\gamma}=m_{L_{\gamma}}$. Since $f^{\dagger} \mid L_{\gamma}=0^{L_{\gamma}}$ we have by induction hypothesis $f \mid L_{\gamma}=f\left(m_{\gamma}\right)^{L_{\gamma}}(\gamma<\rho)$. Since $f$ is continuous and since $f^{\dagger}=0$, it follows by induction on $\gamma<\rho$ that $f\left(m_{\gamma}\right)=0$ for all $\gamma<\rho$, whence also $f(a)=0$. Similarly, let $M_{\delta}=M_{U_{\delta}}$. Then by $f^{\dagger} \mid U_{\delta}=0$ we have by induction $f \mid U_{\delta}=$ $f\left(M_{\delta}\right)^{U_{\delta}}(\delta<\sigma)$. Since $f$ is continuous and $f^{\dagger}=0$ it follows by induction on $\delta<\rho$ that $f\left(M_{\delta}\right)=f\left(M_{K}\right)$ for $\delta<\rho$, whence also $f(a)=f\left(M_{K}\right)$. Thus by $f(a)=0$ we conclude $f=0^{K}$, establishing (ii).

(4) (c) $+(d)+(e) \Rightarrow$ (b). Call an ordered set $B$ a right neighbor extension 
(r.n.e.) of a subset $A \subseteq B$ iff $b \in B-A$ implies $b=\mathrm{mn}_{B}(a)$ for some $a \in A$, or $b=m_{B}$. It is readily checked that if $B$ is an r.n.e. of $A$ then $B$ is compact [scattered] whenever $A$ is compact [scattered] and that $B$ and $A$ are both finite or else $|B|=|A|$.

Let $K$ be a compact ordered set and let $A$ be a closed subset of $K$. For $b \in K-A$ let $\langle b\rangle$ denote the largest open interval in $K$ containing $b$ and disjoint from $A$. Define the reduction of $K$ by $A$ as the ordered set $B=A \cup$ $\{\langle b\rangle: b \in K-A\}$ ordered by the obvious order inherited from $K$. Since $A$ is closed, $B$ is an r.n.e. of $A$ (if $b \in K-A,\langle b\rangle \neq m_{B}$ then $\langle b\rangle=\operatorname{rn}_{B}(\sup (A \cap$ $\left.\left.\left.\left[m_{k}, b\right)\right)\right)\right)$.

Assume now (c) $+(\mathrm{d})+(\mathrm{e})$, and we prove (b).

Let $K$ be a nonempty compact scattered set, and let $f \in C(K)$. Let $A_{0}=\left\{a \in K: f^{\dagger}(a) \neq 0\right\}$, and let $A=\bar{A}_{0}$. Then $A$ is compact, and since $A_{0}$ is a countable subset of $K$, so is $A$ by (d). Let $B$ be the reduction of $K$ by $A$. Then $B$ is again a countable compact ordered set. Let $I$ be the closure in $K$ of some member of $B-A$. Then $(f \mid I)^{\dagger}=0^{I}$ and so, by (c), $f \mid I$ is constant. Thus $b \in K-A, b^{\prime} \in\langle b\rangle$ imply $f(b)=f\left(b^{\prime}\right)$. Define a real function $\hat{f}$ on $B$ by $\hat{f}|A=f| A, \hat{f}(\langle b\rangle)=f(b)$, for $b \in K-A$. By our previous remark, $\hat{f}$ is well defined. It is easily seen that $\hat{f} \in C(B)$, and that $\hat{f}^{\dagger}(a)=f^{\dagger}(a)$ for $a \in A$, $\hat{f}^{\dagger}(\langle b\rangle)=0$ for $b \in K-A$. For $g \in C(B)$ define $\check{g} \in C(K)$ by $\check{g}|A=g| A$ and $\check{g}(b)=g(\langle b\rangle)$ for $b \in K-A$. Then $g \rightarrow \check{g}$ is a linear isometry of $C(B)$ into $C(K)$ carrying $\hat{f}$ to $f$, and $\{\check{J}: J$ is an increment of $\hat{f}\}$ is the set of increments of $f$. Now, by (e), there is an enumeration $\left(J_{n}\right)_{n}$ of $\hat{f}$ s increments, such that $\hat{f}=\Sigma_{n} J_{n}$ holds in $C(B)$. Applying " to both sides, we obtain $f=\Sigma_{n} \check{J}_{n}$ in $C(K)$, and so $f$ equals a sum of its increments.

This completes the proof of Theorem 1 .

CONCLUDING REMARKS. (1) There are scattered compact spaces where (d) holds, that are not orderable (e.g. the one point compactification of an uncountable discrete set). There are also scattered compact Hausdorff spaces where (d) fails (e.g. the one point compactification of the set of all zero-one sequences of length $\leqslant \omega$, topologized by the requirements that each finite sequence is isolated, and that a basic open neighborhood of an infinite sequence $\alpha$ consists of $\alpha$ and all but finitely many of $\alpha$ 's initial segments).

Problem. Classify the (scattered) compact Hausdorff spaces where (d) holds.

(2) Theorem 1 gives an extension of Čech's observation that every continuous real function on $\omega_{1}$ is ultimately constant [C]. In fact, it follows from it that for any continuous real function $f$ on a compact scattered ordered set $K$ there is a closed countable set $A \subseteq K$ such that $f$ is constant on each convex component of $K-A$ (see the proof of (4)).

(3) Theorem 1 stays true if $C(K)$ is replaced by $C(K ; V)$, the space of all continuous functions from $K$ into an arbitrary Banach space $V$ (as (e) is true in this more general context $[\mathbf{M}])$. 
ADDED IN PROOF. The class of compact Hausdorff spaces where (d) holds is also discussed by Mrowka, Rajagopalan and Soundararajan [MRS]. P. R. Meyer showed that (d) is true in every scattered suborderable space (private communication).

\section{REFERENCES}

[BS] C. Bessaga and A. Pelcziński, Spaces of continuous functions. IV, Studia Math. 19 (1960), 53-62. MR $22 \# 3971$.

[C] E. Cech, On bicompact spaces, Ann. of Math. (2) 38 (1937), 823-844.

[HK] A. Haar and D. König, Uber einfach geordinete Mengen, J. Reine Angew. Math. 139 (1911), 16-28.

[K] J. L. Kelley, General topology, Van Nostrand, Toronto, 1964.

[M] G. Moran, Continuous functions on countable compact ordered sets as sums of their increments, Trans. Amer. Math. Soc. (to appear).

[MRS] S. Mrowka, M. Rajagopalan and T. Soundararajan, Chain compact spaces, TOPO-72, General Topology and its Applications, Lecture Notes in Math., vol. 378, Springer, Berlin and New York, 1974, pp. 288-297.

[PS] A. Polczinski and B. Semadeni, Spaces of contimuous functions. III, Studia Math. 18 (1959), 211-222. MR 21 \#6528.

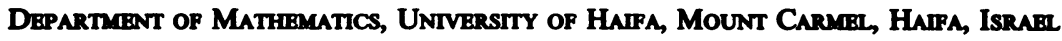

